\title{
In-situ radiography of a split-Hopkinson bar dynamically loaded materials
}

\author{
A. Cohen, ${ }^{a, b, 1}$ D.Levi-Hevroni, ${ }^{a}$ P. Fridman, ${ }^{a}$ D. Chapman,,${ }^{b, c}$ A. Rack, ${ }^{d}$ M.P. Olbinado, ${ }^{d}$ \\ A. Yosef-Hai ${ }^{a}$ and D. Eakins ${ }^{b, c}$ \\ ${ }^{a}$ Department of Physics, NRCN, \\ PO Box 9001, Be'er Sheva, 8419001, Israel \\ ${ }^{b}$ Institute of Shock Physics, Department of Physics, Imperial College, \\ London, SW7 2BW, U.K. \\ ${ }^{c}$ Department of Engineering Science, University of Oxford, \\ Oxford, OX5 IPF, U.K. \\ ${ }^{d}$ ESRF - The European Synchrotron, \\ CS40220, Grenoble, 38043, France \\ E-mail: cohen.amitay@gmail.com
}

Aвstract: This paper presents a description and the demonstration results of a custom-designed bespoke Split-Hopkinson Pressure Bar (SHBP) which has been installed at the ID-19 beamline at the European Synchrotron Radiation Facility (ESRF). Building upon recent advances in realtime $\mathrm{x}$-ray imaging, this system enables the study of dynamic mechanical phenomena through ultra-high-speed x-ray phase-contrast radiographs captured every $528 \mathrm{~ns}$ with high spatial-temporal resolution. By adding synchronized strain gauges measurements in the same experiment, bulk stress-strain behaviour can be correlated to the local processes underlying deformation, damage and failure. This article briefly outlines the newly installed hardware and its design. Demonstration experiments showing damage development in dynamically loaded Ti-6Al-4V and concrete are presented to underline the potential of an SHBP to be used in combination with synchrotron-based high-speed hard $\mathrm{x}$-ray imaging.

KeYwords: Inspection with x-rays; X-ray radiography and digital radiography (DR); Trigger concepts and systems (hardware and software)

\footnotetext{
${ }^{1}$ Corresponding author.
} 


\section{Contents}

1 Introduction 1

2 SHPB design and real-time X-ray imaging 1

3 Experiments 3

4 Summary 5

\section{Introduction}

The Split-Hopkinson Pressure Bar (SHPB) is a well-known experimental platform for measuring the mechanical response of materials subjected to high strain rates [1]. Although this technique has existed in various forms for over a century [2], it is still relied upon for the characterisation of new material compounds and associated fabrication methods, as well as for the fundamental studies of dynamic fracture and failure. A new approach which involves in-situ, fast x-ray imaging of SHPB experiments was presented recently by Chen et al. [3, 4]. The main benefit of such a technique is the ability to observe the interior of the tested material and to follow plastic deformation and failure behaviour of dynamically loaded opaque materials. This paper presents a complementary, custom-made SHPB system that was set up at the ID-19 beamline at the European Synchrotron Radiation Facility (ESRF). Thanks to the flexibility of the system it can be easily installed in the hutch and can accommodate various types of Hopkinson Bar experiments such as conventional SHPB, mini SHPB for higher strain rates and direct impact tests. All versions are equipped with conventional strain gauges ( $\mathrm{SG}$ ) in order to match the loading signal's history to real-time $\mathrm{x}$-ray imaging. Large beam diameters up to the "centimetre-range", and long propagation distance phasecontrast imaging for studying mesoscale objects are key parameters for high lateral resolution and sensitivity combined with a relatively large field of view. This setup at ID-19 is accessible to the public within the standard user program of the ESRF.

\section{SHPB design and real-time X-ray imaging}

The SHPB system is designed specially to accommodate the ID-19 hutch space and to allow convenient and effective working. In order to place the sample in front of the x-ray beam with the hutch room limitations (figure 1), bars were cut to length: $1300 \mathrm{~mm}$ input bar and $1100 \mathrm{~mm}$ output bar, $12.7 \mathrm{~mm}$ diameter. The projectiles are 400,250 and $150 \mathrm{~mm}$. The system base frame is height adjustable to follow the beam's changeable height (monochromatic or pink beam and because of a seasonal effect). Bars and projectiles are made of PH 17-4 steel with the H900 thermal treatment which brings the yield strength to $1.2 \mathrm{GPa}$ and sound velocity $5020 \mathrm{~m} / \mathrm{s}$ (measured on the system). A set of protective anvils was designed to enable a wide variety of materials to be tested (such 


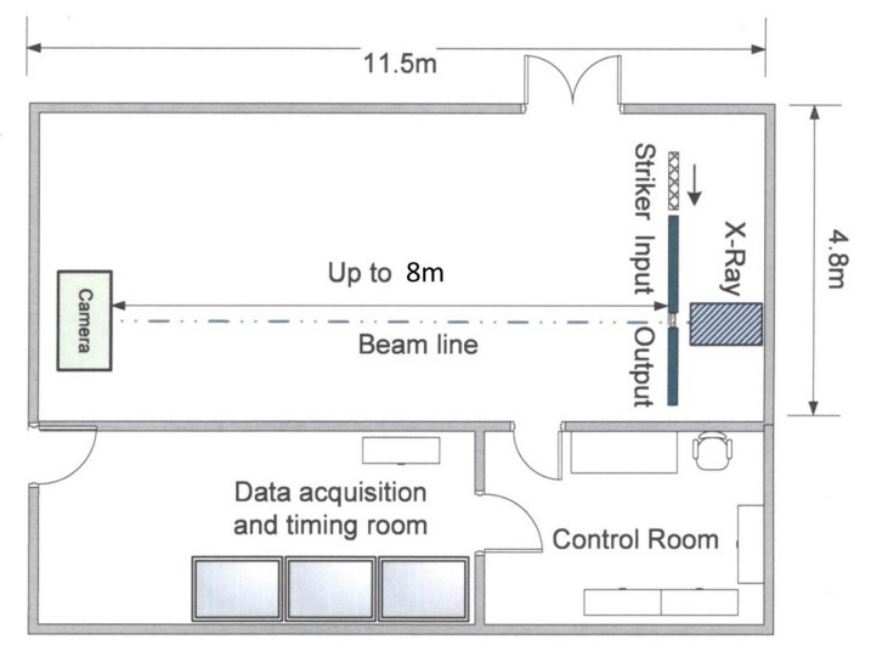

Figure 1. ID19 Hutch and control room scheme. The dimensions of the SHPB system are appropriate for the hutch room. Long propagation distance phase-contrast imaging $(8 \mathrm{~m})$ ensures a high sensitivity for cracks.

as hard metals, concrete and $\mathrm{Al}_{2} \mathrm{O}_{3}$ ). A launching system based on a simple gas gun, is remotely controlled from the hutch control room and we succeeded in minimising the risk of false triggers. The gun operation pressure range is $0.5-15$ bar which creates a velocity range of $4 \mathrm{~m} / \mathrm{s}$ up to $\sim 35$ $\mathrm{m} / \mathrm{s}$. A protective case, with virtually x-ray clear windows made of thin Mylar film, encapsulates the sample and its fragments, ensuring that no damage occurs to the equipment in the hutch. A removable marking needle is located on a kinematic mount in the centre of the case in order to locate the $\mathrm{x}$-ray beam and the image relative to the sample centre.

Ultra-high time-resolution is achieved by means of single-bunch imaging: we used the ESRF operating in 4-bunch or 16-bunch mode, i.e. 4 or 16 bunches of light source are delivering a $\sim 100$ ps x-ray flash every $704 \mathrm{~ns}(1.4 \mathrm{MHz}$ repetition rate) or $176 \mathrm{~ns}(5.7 \mathrm{MHz})$ respectively; this is short enough to "freeze"/capture ultra-fast motion such as crack propagation in the pictures [5, 6]. A wide field of view (up to $\sim 20 \times 10 \mathrm{~mm}^{2}$, horizontal $\times$ vertical) enables observing a whole sample with one image. An indirect detector consisting of an LYSO:Ce single-crystal scintillator (40 ns delay) combined with $1 \times$ and $4 \times$ magnifying optics ( 0.2 numerical aperture) relays the luminescence corresponding to each transmitted $x$-ray pulse onto the sensor of a Shimadzu HPV-X2 camera. In the work presented in this paper we mainly used a $528 \mathrm{~ns}$ inter-frame temporal sampling. The intermediate storage of the camera of 128 frames allows one to acquire a sequential series of images ("movies") over a total duration of nearly $68 \mu$ s (figure 2). In some cases we integrated 3 bunches in one exposure per frame to enhance the flux per image; $880 \mathrm{~ns}$ inter-frame temporal sampling was used (580 ns exposure time) which leads to nearly $113 \mu$ s duration. Recent detector developments underlined that all bunches can be exploited in 16 bunch mode when up to three cameras are combined, i.e. the full pulse rate of $5.7 \mathrm{MHz}$ can be exploited for imaging [7]. A long propagation distance phase-contrast $(8 \mathrm{~m})$ ensures a high sensitivity for cracks, i.e. not limited by the pixel size of the detector. The beamline's two U-32 type undulators, set to the minimal gap, deliver the required photon flux density [5]. 


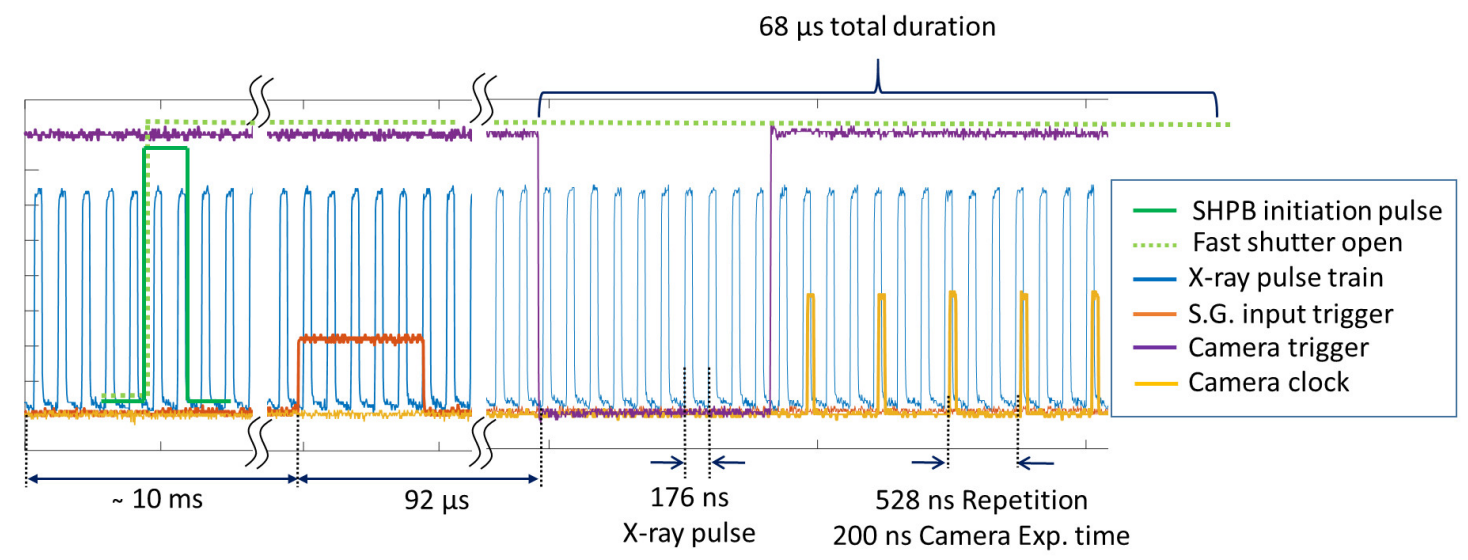

Figure 2. Synchronization of SHPB, SG signals, bunch clock and camera trigger. The SHBP "Shot" and "Fast shutter open" signals were not measured and hence added manually.

Firing is accomplished remotely from the ID-19 control room in the following manner: a "shooting" command synchronized with the x-ray pulses is generated using a coincidence logic unit, a bunch clock delay unit and a computer triggered signal [5]. The "shooting" command starts the operation and simultaneously both fires the SHPB system and opens the x-ray hutch fast shutter. A delay generator controls the needed time interval between an input trigger signal (incident signal which presents the loading pulse), the camera exposure time and the x-ray pulse train. The specific delay is calculated to ensure the desired camera triggering time relative to the actual sample loading duration and to the x-ray pulse train. A single event, the incident signal on the input bar, triggers two separate DAC scopes. One oscilloscope measures the SHPB data (projectile velocity and strains on the bars) and a second oscilloscope presents the bunch clock signal (x-ray pulse train), camera trigger and camera (frames) clock (figure 3). This sequence creates a trustable control system and puts all signals on two matched time axes for reliable data analysis. The sequence also allows matching conventional SHPB strain gauges signals with $\mathrm{x}$-ray dynamic radiography.

\section{Experiments}

Dynamic loading and radiography were applied in ID-19 on a series of Additive Manufacturing Electron Beam Melting (AM-EBM) Ti-6Al-4V. Specimens were compressed under shear load condition using Modified Shear Compression Specimen (MSCS) geometry [8]. The specific geometry of the sample, which was designed for large shear strain at the centre of the gauge, enabled us to zoom-in to the shear localization area. A $0.5 \mathrm{~mm}$ gauge thickness strikes a balance between mechanical stability and $x$-ray transparency (figure 4). In the experiment presented in this paper we used $4 \times$ magnification and captured an area of $2 \times 3 \mathrm{~mm}^{2}$ at the gauge centre.

The crack propagation pattern matched the stress loading signals as shown in figure 5 [9]. A common time axis of the strain gauges and of the above described camera, shows a match between the first failure pattern observation in figure 5(a). The pattern is marked with a dashed line. The pattern appeared just after the end of the maximum load point which was measured on the transmitted bar, shown in figure 5(c). In figure 5(b), multiple cracks can be seen in the gauge centre. For the sake of simplicity, we took the beginning of the sample load, i.e. when the loading 


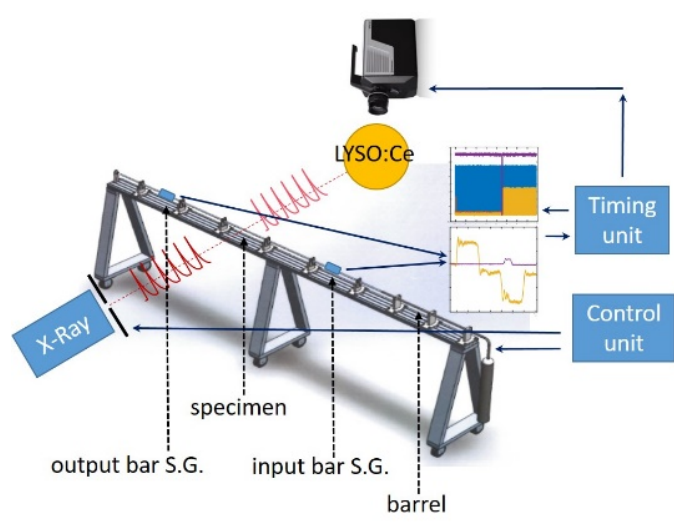

(a)

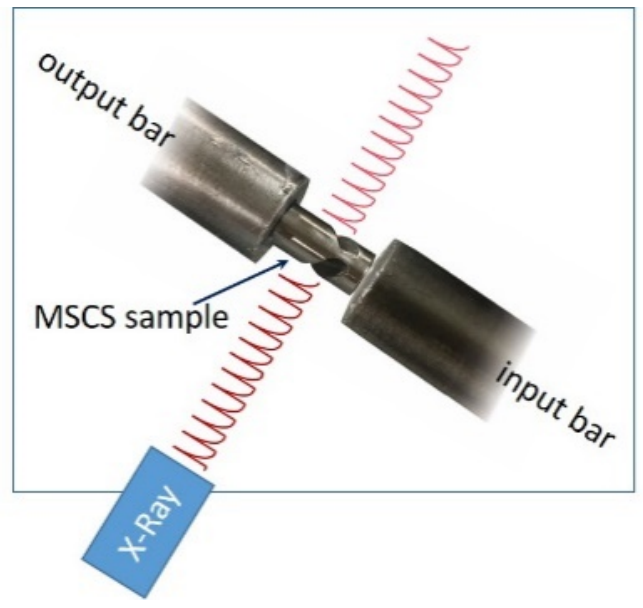

(b)

Figure 3. (a) SHPB setup mounted perpendicular to the X-ray axis in ID19. A shooting pulse from the control unit opens the fast shutter and launches the SHPB projectile. SG signal (measured on bars) sent to the timing unit and after the desired delay, triggers the fast camera. Dynamic radiography of the loaded sample is converted to visible light by LYSO:Ce scintillator and captured on fast camera. The SHPB base is rolling and the height is adjustable which enable locating the sample at the beam centre. (b) Sample zoom-in. Top view of Modified Shear Compression Specimen (MSCS) sample mounted between input and output bars, sample centre and beam centre are matched.

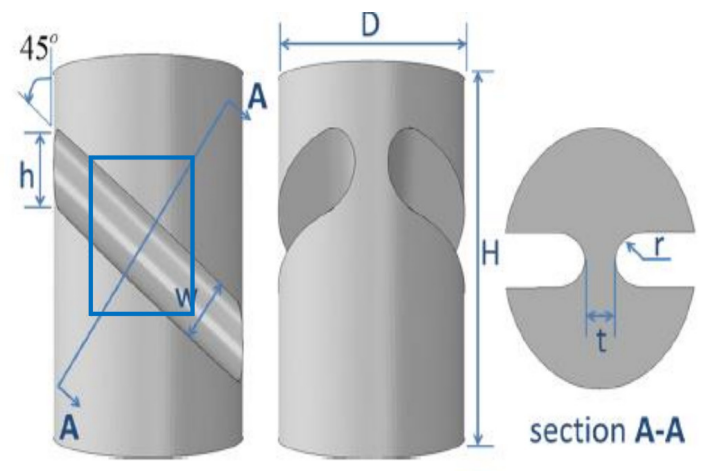

Figure 4. MSCS sample (reprinted from [8] with permission). The thickness of the gauge, $t=0.5 \mathrm{~mm}$, enables mechanical stability and X-ray transparency. The width, $\mathrm{w}=1 \mathrm{~mm}$ radius, ensures well-defined shear localization area, easily zoomed-in. Blue rectangle on the left sketch represents the area which is shown in figure 5 .

pulse reaches the input bar's front surface, as $t=0$. Hence, the time of the image in figure $3(\mathrm{a})$ is $t=74.1 \mu \mathrm{s}$ from the beginning of loading and the time of the image in figure $5(\mathrm{~b})$ is $t=86.5 \mu \mathrm{s}$. This corresponds to time point just before the end of the measured load. In this measurement we used $580 \mathrm{~ns}$ exposure time to capture 3 x-ray bunches in each frame and the temporal uncertainty in that case is $\pm 290 \mathrm{~ns}$. The widths of the first and second parts of the transmitted signal falling edge are $12.9 \mu$ s and $10.4 \mu$ s respectively, as shown in figure 5(c). This brings the temporal matching uncertainty to less the $3 \%$ for each falling section. A $4 \times$ magnification was used in this case. 

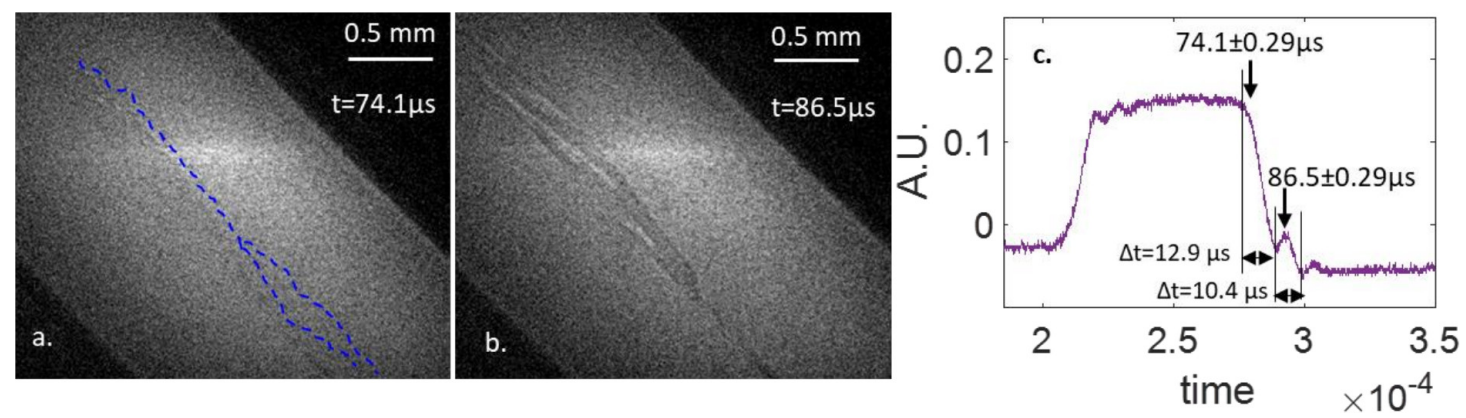

Figure 5. Failure of Ti-6-4 (EBM type): (a) First observation of damage (dashed marked) at $t=74.1 \mu \mathrm{s}$. (b) Significant fractures on the centre of gauge at $\mathrm{t}=86.5 \mu \mathrm{s}$. (c) Load signal measured on transmitted SG with corresponding arrow marks of sub-figures (a) \& (b) time. Note that the time axis belongs to the measurement while the arrows marking the time correspond to the images.
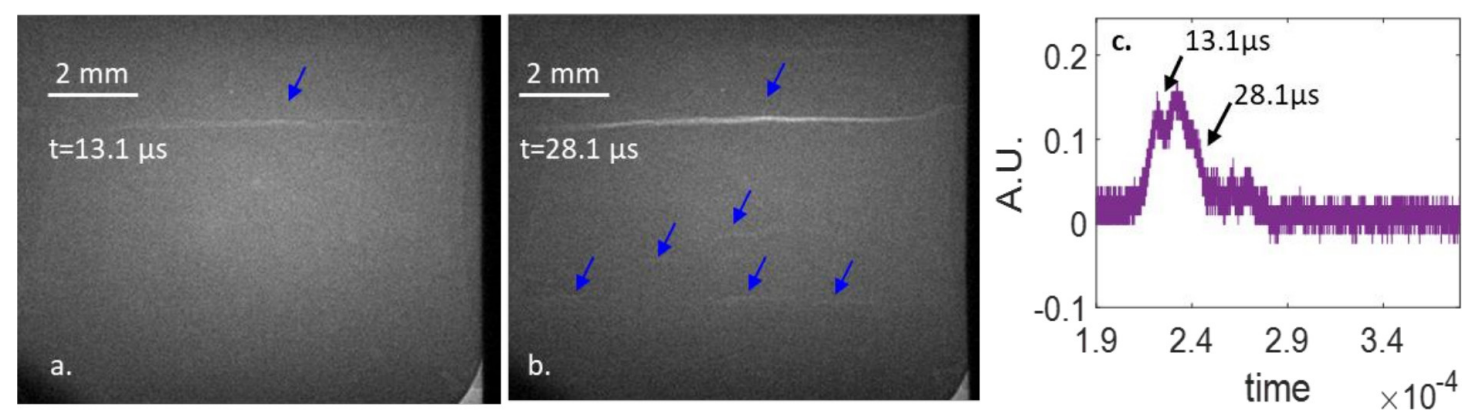

Figure 6. Failure of concrete cement: (a) First observation of damage (light grey line) matches the first sign of damage at the transmitted SG signal, $t=13.1 \mu \mathrm{s}$. (b) Multiple cracks observed at the sample radiography, $t=28.1 \mu$ s. (c) Load signal measured on transmitted SG with corresponding arrow marks of (a) \& (b) time.

The failure mode of concrete cement with no aggregates is demonstrated in figure 6 . The samples were cast into $10 \times 10 \mathrm{~mm}^{2}$ square and polished to $3 \mathrm{~mm}$ thick. A $1 \mathrm{~mm}$ radius on 4 corners of the sample was added on the casting mould to reduce stress concentration on sharp edges. The load was applied on the $10 \mathrm{~mm}$ axis with the $3 \mathrm{~mm}$ wall thickness perpendicular to the $\mathrm{x}$-ray beam. Two thin stainless-steel anvils were mounted on the bars to protect the bars' surfaces without any impedance mismatch. A $1 \times$ magnification was used in this experiment and a field of view of $10 \times 8$ $\mathrm{mm}^{2}$ was set to capture the whole sample. Figure 6(a) shows the first failure pattern at $t=13.1 \mu \mathrm{s}$. In figure 6(b) you can see multiple cracks at $t=28.1 \mu \mathrm{s}$. The signal measured on the output bar is shown in figure $6(\mathrm{c})$. The corresponding time for figures $6 \mathrm{a} \& 6 \mathrm{~b}$ is marked with a black arrow. An exposure time of $200 \mathrm{~ns}$ was used which leads to a temporal uncertainty of $\pm 100 \mathrm{~ns}$.

\section{Summary}

We have reported the design and installation of an SHBP system at beamline ID-19 of the ESRF. The setup has been successfully commissioned and is now accessible to the public via the user programme of the ESRF. The benefit of in-situ, dynamic, radiography measurements, combined with conventional load signals measured on the SHPB bars, is clearly presented in two demonstration experiments. This combined approach emphasises the additional information which can be extracted 
from matching the failure process to the conventional SHPB signals. Information such as this can be used in material failure modelling and in future investigation of fracture behaviour, complex material and material strength of the products of new manufacturing processes.

Future work will include extending the loading capabilities with an additional tension bar. The new ESRF brilliant light source [10] program (EBS) will hopefully help as to achieve a dynamic diffraction diagnostic and give us the option of tracing structural dynamic phenomena such as dynamic recrystallization (DRX) or even phase transition in higher strain rate regime.

\section{Acknowledgments}

The authors would like to thank Prof. A. Frydman (BIU) for supporting the system setup process. Technical help from the main mechanical workshop - NRCN, is acknowledged. Ti-6Al-4V samples were kindly provided by M. Chonin, AM Centre, Rotem Ltd. Concrete samples were kindly provided by Dr G. Tear, ISP-ICL. We would like to thank Dr L. Farbaniec, ISP-ICL for useful discussion. Assistance with graphic illustrations by T. Harpenes is acknowledged. European Synchrotron Radiation Facility granted beamtime in the frame of long-term proposal MI1252. This research was funded by the Israel Atomic Energy Commission (NRCN-SHPB), Defence Science and Technology Laboratory (DE), Engineering and Physical Sciences Research Council (DC), Atomic Weapons Establishment.

\section{References}

[1] B.A. Gama, S.L. Lopatnikov and J.W. Gillespie Jr., Hopkinson bar experimental technique: a critical review, Appl. Mech. Rev. 57 (2004) 223.

[2] B. Hopkinson, A method of measuring the pressure produced in the detonation of high explosives or by the impact of bullets, Phil. Trans. Roy. Soc. London A 213 (1914) 437.

[3] W.W. Chen et al., In-situ damage assessment using synchrotron X-rays in materials loaded by a Hopkinson bar, Phil. Trans. Roy. Soc. London A 372 (2014) 2013.

[4] M.C. Hudspeth et al., High speed synchrotron x-ray phase contrast imaging of dynamic material response to split Hopkinson bar loading, Rev. Sci. Instrum. 84 (2013) 025102.

[5] A. Rack et al., Exploiting coherence for real-time studies by single-bunch imaging, J. Synchrotron Radiat. 21 (2014) 815.

[6] M.P. Olbinado et al., Ultra high speed $x$-ray imaging of laser-driven shock compression using synchrotron light, J. Phys. D 51 (2018) 055601.

[7] E.M. Escauriza et al, Ultra-high-speed indirect x-ray imaging system with versatile spatiotemporal sampling capabilities, Appl. Opt. 57 (2018) 5004.

[8] A. Dorogoy, D. Rittel and A. Godinger, Modification of the shear-compression specimen for large strain testing, Exp. Mech. 55 (2015) 1627.

[9] J. Schindelin et al., Fiji: an open-source platform for biological-image analysis, Nature Meth. 9 (2012) 676.

[10] M.P. Olbinado et al. , MHz frame rate hard X-ray phase-contrast imaging using synchrotron radiation Opt. Expr. 25 (2017) 13857. 